\title{
Notes sur la spécificité, la localisation et la chorologie des Anoploures et de quelques autres Insectes ectoparasites de micromammifères en France
}

\author{
Par J.-C. BEAUCOURNU et B. RAULT * \\ [Laboratoire de Parasitologie et Zoologie appliquée (professeur: J.-M. DoвY), \\ Faculté de Médecine et de Pharmacie de Rennes]
}

\begin{abstract}
Résumé
L'étude des ectoparasites de 8.000 petits Mammifères a montré quelques faits intéressants concernant leur écologie et particulièrement leur spécificité (plus ou moins stricte suivant les espèces), leur localisation sur l'hôte (variable en fonction du parasite considéré) et leur chorologie qui, souvent, obéit à d'autres facteurs que la simple superposition à la répartition géographique de l'hôte principal.
\end{abstract}

\section{Summary}

The study of 8.000 small Mammals' ectoparasites showed some interesting facts concerning their ecology and particularly their specificity (more or less strict according to the species), their localization on the host (varying with the considered parasite) and their chorology, which often depends of other factors than the simple superposition on the principal host's geographic repartition.

(*) Réunion de la Société Française de Parasitologie, Montpellier, mai 1967. 
Ces notes, basées sur l'examen de 8.000 petits Mammifères, plus de 3.000 étant des terrestres et les autres des Chiroptères, voudraient souligner quelques points de ce que nous croyons savoir sur la spécificité, la localisation et la chorologie de leurs ectoparasites et en particulier des poux.

\section{A. - Spécificité :}

Bien que nous soyons convaincus de la spécificité élevée de l'immense majorité des ectoparasites, il faut souligner que beaucoup d'entre eux, inféodés à une espèce d'hôte, le sont peut-être par « obligation». Beaucoup de genres de micromammifères sont en effet représentés par une seule espèce dans la zone qui nous occupe.

C'est ainsi que l'Anoploure Hoplopleura longula (Neumann) ne se rencontre que sur le rat des moissons Micromys minutus (Pallas), H. captiosa Johnson ne parasite que la souris Mus musculus L., mais, en fait, dans un cas comme dans l'autre, aucun hôte du même genre ne vit en Europe. Par contre, si le genre-hôte comprend plusieurs espèces dans une même zone, il n'est pas rare que le parasite élargisse son champ d'action. Ainsi Polypax spinigera (Burmeister), pou des Arvicola (rats d'eau), se rencontre sur A. amphibius L., A. sapidus Miller, A. terrestris L. et même $A$. scherman Shaw si l'on admet la validité spécifique de ce taxon. De même, $P$. borealis Ferris parasite plusieurs espèces du genre Clethrionomys...

Dans le même ordre d'idée, citons le Coléoptère Leptinus testaceus Müller, les puces Leptopsylla taschenbergi Wagner et Typhloceras poppei Wagner sur le genre Apodemus, le Diptère Nyctéribiidé Nycteribia biarticulata Hermann sur les espèces du genre Rhinolophus...

Il faut même admettre, dans l'état actuel de nos connaissances, que certains Anoploures, Insectes cependant très spécifiques, peuvent se rencontrer sur deux genres [Polyplax serrata (Burmeister) sur mulots et souris], ou plus $[P$. reclinata (Mitzsch), pou de musaraignes, considéré, à tort ou à raison, comme monotypique, est signalé de tout l'Ancien Monde sur plus de quinze espèces appartenant à trois sous-familles différentes (Beaucournu et Houin 1967)].

Inversement, il existe de réels cas de dépendances étroites entre hôte et parasite, les uns comme les autres appartenant pourtant à des genres représentés par plusieurs espèces à localisation géographique commune. On peut citer les Mallophages de Mustelidae (Trichodectes s. g. Stachiella) (Hopkins 1941), ou encore les Diptères du sous-genre Nycteribia inféodés au genre Myotis (Theodor 1954, Aellen 1955, Beaucournu 1961, 1962) :

Nycteribia pedicularia Latreille vit sur M. capacinii (Bonaparte),

Nycteribia latreillei Leach sur M. myotis (Bechstein),

Nycteribia kolenatii Theodor sur $M$. daubentoni (Leisler).

Enfin, mentionnons le cas des deux taupes frasçaises Talpa europaea L. et $T$. caeca Savi souvent peu faciles à distinguer, mais que l'examen de leurs puces dissocie parfaitement, $T$. europaea étant parasitée, en France, par Palaeopsylla minor Dale et $T$. caeca par $P$. cisalpina Jordan et Rothschild (Beaucournu, sous presse). 
Des exemples aussi tranchés ne sont pas connus en France chez les Anoploures de petits Mammifères, dans l'état actuel de la taxonomie. Nous pouvons toutefois signaler, à propos de plus grandes espèces, le cas des Suidés. Nous avons, en France, deux représentants du genre Sus. S. domesticus L., le porc domestique, héberge Haematopinus suis (L.), S. scrofa L., le sanglier, est parasité par $H$. apri Goureau.

De quelle nature est cette spécificité ? Phylogénique certainement, chez beaucoup de Siphonaptères, les Anoploures, et d'une façon très remarquable chez les Mallophages (Hopkins $1957 a, 1957 b$ ). Elle est, sans aucun doute, écologique chez la plupart des puces des genres Ctenophthalmus, Hystrichopsylla, etc... De nombreux cas, toutefois, sont encore douteux.

\section{B. - Localisation :}

Il est bien connu que l'homme offre trois différents écoumènes en ce qui concerne les poux : les cheveux sont habituellement le domaine de Pediculus capitis, le corps, son revêtement pileux fin et les vêtements qui le recouvrent celui de $P$. corporis, les poils pubiens, enfin, celui de Phthirus pubis. De semblables localisations existent pour de très nombreux parasites de petits (et de grands) Mammifères.

C'est ainsi que, en ce qui concerne les Anoploures, Polyplax serrata, pou du mulot, se rencontre surtout au niveau des épaules et de la région interscapulaire, $P$. hannswrangeli Eichler, parasite du campagnol roussâtre Clethrionomys glareolus (Schreber) sur les flancs, le pou du lapin Haematopinus ventricosus (Denny) autour des mammelles et Enderleinellus nitzschi Fahrenholz, inféodé à Sciurus vulgaris L., l'écureuil, uniquement au niveau du creux axillaire (Waterson 1912; Beaucournu 1967).

$\mathrm{Si}$ nous considérons d'autres ectoparasites, on peut noter les localisations préférentielles suivantes :

- Le Coléoptère Leptinus testaceus à la face dorsale de la racine de la queue (1).

- Echidnophaga murina (Tirab.) (Siphonaptère) parasite des Muridés, sur la tête, au niveau des mâchoires inférieures surtout (Tiraboschi 1903, 1904 ; Beaucournu et coll. 1965).

- Spilopsyllus cuniculi (Dale), puce du lapin, sur les oreilles (Allan 1956).

De même chez de grands Mammifères:

- Chez le chevreuil, le Mallophage Damalinia meyeri (Taschenberg) est particulièrement abondant au niveau du périnée.

- La mouche des Cervidés, Lipoptena cervi (L.) a également de telles niches écologiques: près de $50 \%$ de ces parasites sont récoltés sur le cou ou aux aines (Haarlov 1964).

Il est certain que de telles localisations répondent à des besoins précis exigés, soit par la morphologie du parasite, soit par un microclimat particulier, soit enfin

(1) Ceci, à notre sens, étant un argument en faveur de l'opinion qui fait de Leptinus un parasite vrai et non un phorétique. 
par la recherche, tout simplement, de zones à peau plus fine, cela étant alors en rapport avec des pièces buccales peu développées. Enfin, les régions du corps que l'hôte ne peut que difficilement gratter peuvent être, de ce fait même, plus recherchées. Ce dernier cas semble cependant moins fréquent qu'on ne le croirait.

\section{C. - Chorologie :}

Schématiquement, on peut admettre qu'un ectoparasite spécifique suit la répartition de son hôte: c'est ainsi que le pou Polyplax spinulosa a suivi le rat d'un bout à l'autre du globe ; c'est ainsi que les trois Anoploures [Enderleinellus longiceps $\mathrm{K}$. et F., Hoplopleura sciuricola F. et Neohaematopinus sciurinus (Mjöberg)] et la puce [Orchopeas h. howardi (Baker)] inféodés à l'écureuil gris d'Amérique du Nord, Sciurus carolinensis, se sont acclimatés avec leur hôte en Grande-Bretagne, maintenant, semble-t-il, une fréquence et une densité notables. Cependant, certaines espèces, bien que capables de subir des climats très variés, présentent localement des taux extrêmement variables. Haemodipsus ventricosus, le pou du lapin, a suivi son hôte partout où il fut introduit ; mais, même sous des climats comparables, sa fréquence peut être très inégale : 134 hôtes sur 374 sont trouvés parasités en Grande-Bretagne par Mead-Briggs et Hughes (1965). A peu près à la même époque, dans l'Ouest de la France, nous ne trouvons aucun lapin parasité sur 50 .

Dans d'autres cas, le parasite est plus sensible que son hôte aux facteurs climatiques. Il ne pourra alors s'adapter aux zones extrêmes colonisées par son hôte à moins de microclimats insolites sous ces latitudes. Cela est particulièrement net pour les parasites ayant un stade libre, les puces par exemple. C'est ainsi que Pulex irritans L. ne parasite pas les peuples de l'extrême nord (alors que les poux surabondent!); Xenopsylla cheopis (Roths.), puce de rats, d'origine très certainement nord-éthiopienne ou proche asiatique ne peut se maintenir sous nos climats que dans des conditions particulières, essentiellement élévation de la température ambiante: sous-sols d'immeubles et, dans un cas suivi par Roman, galeries de mine (Roubaud 1928, Roman 1938, Roman et Puech 1965) (2). Elle ne se rencontre pas. par contre, sur les hôtes capturés à distance des ensembles urbains ou industriels.

De par sa situation privilégiée, la France présente toutefois dans sa faune naturelle aussi bien des éléments boréo-alpins [les puces Amphipsylla sibirica (Wagner), plusieurs Ceratophyllus, vraisemblablement le pou Neohaematopinus sciuri Jancke] que des éléments éthiopiens [les puces Araeopsylla gestroi (Roths.), Stenoponia tripectinata, etc...]. Cependant, les premiers ne se rencontreront chez nous qu'en altitude, les seconds, que dans le midi méditerranéen.

Un exemple assez frappant concernant les éléments boréo-alpins nous semble donné par les poux du campagnol roussâtre Clethrionomys glareolus. Ayant capturé des centaines de ces Rongeurs dans l'Ouest et le Nord, nous avions acquis la conviction

(2) Nous ne pouvons admettre la théorie suivant laquelle $X$. cheopis aurait été le principal vecteur européen au cours des grandes pandémies pesteuses historiques (Hirst 1923, Girard 1946...). Ce rôle fut certainement tenu, dans la transmission interhumaine, par $P$. irritans dont Blanc et Baltazard (1945) ont souligné l'importance épidémiologique. 
qu'ils n'hébergeaient jamais d'Anoploures. Or, nos collègues d'Europe Centrale et Orientale les donnaient au contraire comme très parasités, ce que nous avions pu, d'ailleurs, constater par nous-même en Pologne. Des piégeages dans la région alpine française et le Massif Central nous ont, localement, dans des zones coïncidant parfaitement jusqu'à présent avec les limites d'extension des glaciers quaternaires, montré des populations de Clethrionomys très infestées hébergeant deux poux hautement spécifiques, Polypax hannswrangeli et Hoplopleuira edentula. Cette dernière espèce était, malgré une ancienne description de Fahrenholz (1916), considérée comme identique à celle, banale et largement répandue, parasitant les campagnols du genre Microtus. Ce fut cette discordance entre la présence localisée de poux du genre Hoplopleura sur Clethrionomys, et l'ubiquité de l'Hoplopleura de Microtus qui nous permit de constater la validité de $H$. edentula (Beaucournu 1966).

Ceite dépendance, vis-à-vis du climat, d'ectoparasites aussi liés à leurs hôtes que les Anoploures, nous paraît intéressante. Ce n'est là, toutefois, que l'une des multiples voies de recherche que nous ouvre l'étude de l'écologie des ectoparasites et dont nous avons tenté de montrer brièvement quelques aspects.

\section{Bibliographie}

Aellen (V.), 1960. - Notes sur les puces des chauves-souris, principalement de la Suisse (Siphonaptera, 1schnopsyllidae). Bull. Soc. Neuchat. Sc. Nat., 83, 41.

Allan (R. M.), 1956. - A study of the populations of the rabbit flea Spilopsyllus cuniculi (Dale) on the wild rabbit Oryctolagus cuniculus in Norih-East Scotland. Proc. $R$. Ent. Soc. Lond. $(A), 31,145$.

Beaucournu (J.-C.), 1961. - Ectoparasites des Chiroptères de l'Ouest de la France. $1^{\text {re }}$ partie : Ixodoïdés, Cimicidés et Nyctéribiidés. Bull. Soc. Scient. Bretagne, 36, 315.

-, 1962. - Nouvelles captures de Nycteribiidae (Diptera, Pupipara) en France. Ann. Par. Hum. et comp. 37, 366.

-, 1966. - Hopopleura edentula Fahrenholz, 1916 (Anoplura), parasite spécifique de Clethrionomys glareolus, est une bonne espèce. Acta Parasitologica Polonica, 14, 127.

—, 1967. - Les Anoploures de Lagomorphes, Rongeurs et Insectivores dans la région paléarctique occidentale et en particulier en France. Ann. Par. Hum. et comp., 42, sous presse.

—, 1968. - Catalogue provisoire des Siphonaptères de la faune française. Bull. Soc. Entom. France, sous presse.

— et Houin (R.), 1967. - A propos de la présence à Madagascar de Polyplax reclinata (Nitzsch 1864) sensu Johnson 1960 (Insecta, Anoplura), parasite des musaraignes. Arch. Inst. Pasteur Madagascar, 36, 67.

-, Rault (B.) et Beaucournu-Saguez (F.), 1965. - Contribution à l'étude des siphonaptères de la Corse. Bull. Soc. Zool. France, 89, 737.

Blanc (G.) et Bal.tazard (M.), 1945. - Recherches sur le mode de transmission naturelle de la peste bubonique et septicémique. Arch. Inst. Pasteur Maroc, 3, 173. 
Girard (G.), 1946. - Présence de Xenopsylla cheopis sur des rats noirs capturés à Paris. Bull, Soc. Path. exot., 39, 355.

HAARlov (N.), 1964. - The distributional pattern of Lipoptena cervi on Danish red deer [Cervus elaphus (L.)] and fallow deer [Dama dama (L.)]. Proc. XII Int. Congr. Ent., London, 788.

Hirst (L.-F.), 1923. - Cité par Pollitzer (R.), La Peste, 1954, Monographie O.M.S., $\mathrm{n}^{\circ} 22$.

Hopkins (G. H. E.), 1941. - Stray notes on Mallophaga III. Ann. Mag. nat. Hist., série $11,7,38$.

-, 1957 a. - Host-associations of Siphonaptera. Premier Symposium sur la spécificité parasitaire des parasites de Vertébrés, U.I.S.B., Paris.

- 1957 b. - The distribution of Phthiraptera on mammals. Premier Symposium sur la spécificité parasitaire des parasites de Vertébrés, U.I.S.B., Paris.

Mead-Brigas (A. R.) et Hughes (A. M.), 1965. - Records of mites and lice from wild rabbits collected throughout Great-Britain. Ann. Mag. Nat. Hist., ser. 13, 8, 695.

Roman (E.), 1938. - Présence à Saint-Etienne de la puce Xenopsylla cheopis chez les rats de mine. Bull. Soc. Zool. France, 63, 302.

- et Puech (G.), 1965. - La puce Xenopsylla cheopis s'est acclimatée à Saint-Etienne dans des galeries de mine. Bull. Soc. Path. exot., 58, 1.103.

Roubaud (E.), 1928. - Foyer de développement de Xenopsylla cheopis à Paris. Observations sur la biologie de cette puce. Bull. Soc. Path. exot., 21, 227.

THÉodor (O.), 1954. - Nycteribiidae, in E. Lindner, Flieg. Palearkt. Reg., 66 a.

Tiraboschi (C.), 1903. - La chique des oiseaux (Sarcopsylla gallinacea Westw.) observée en Europe. Arch. Parasitologie, 7, 124.

-, 1904. - Les rats, les souris et leurs parasites cutanés dans leurs rapports avec la propagation de la peste bubonique. Arch. Parasitologie, 8, 161.

Waterston (J.), 1912. - Haematopinus (Haemodipsus, Enderlein) ventricosus Denny in N. Mavine with note on an easy method of its detection. Ent. mon. Mag., 23, ( $2^{*}$ série), 116. 